\title{
Regional and Hemispheric Variation in Cortical Thickness in Chimpanzees (Pan troglodytes)
}

\author{
William D. Hopkins ${ }^{1,2}$ and Brian B. Avants ${ }^{3}$ \\ ${ }^{1}$ Division on of Developmental and Cognitive Neuroscience, Yerkes National Primate Research Center, Atlanta, Georgia 30322, ${ }^{2}$ Neuroscience Institute and \\ Language Research Center, Georgia State University, Atlanta, Georgia 30302, and ${ }^{3}$ Department of Radiology, University of Pennsylvania, Philadelphia, \\ Pennsylvania 19104-6389
}

Recent advances in structural magnetic resonance imaging technology and analysis now allows for accurate in vivo measurement of cortical thickness, an important aspect of cortical organization that has historically only been conducted on postmortem brains. In this study, for the first time, we examined regional and lateralized cortical thickness in a sample of 71 chimpanzees for comparison with previously reported findings in humans. We also measured gray and white matter volumes for each subject. The results indicated that chimpanzees showed significant regional variation in cortical thickness with lower values in primary motor and sensory cortex compared with association cortex. Furthermore, chimpanzees showed significant rightward asymmetries in cortical thickness for a number of regions of interest throughout the cortex and leftward asymmetries in white but not gray matter volume. We also found that total and region-specific cortical thickness was significantly negatively correlated with white matter volume. Thus, chimpanzees with greater white matter volumes had thinner cortical thickness. The collective findings are discussed within the context of previous findings in humans and theories on the evolution of cortical organization and lateralization in primates.

\section{Introduction}

In modern structural brain imaging protocols, cortical volume $(\mathrm{CV})$ is a main measure of interest from the standpoint of understanding disease progression, development, and aging. CV is made up of cortical thickness, surface area, and the white matter (WM) that underlies connectivity between regions. In terms of brain evolution, the human brain is approximately three times larger than that of chimpanzees, the closest living relative to humans (Rilling and Insel, 1999b; Semendeferi and Damasio, 2000; Semendeferi et al., 2001; Schoenemann, 2006; Sherwood et al., 2012). In addition, humans have disproportionate increases in gray and particularly white matter volume compared with other primates (Rilling and Insel, 1999a; Schoenemann et al., 2005; Rogers et al., 2010). It has been suggested that the relative increase in white matter volume and gyrification are prominent in brain regions, such as the prefrontal cortex (PFC) and temporal lobes, that are thought to underlie certain human cognitive specializations (Deacon, 1997; Semendeferi et al., 1998; Rilling and Seligman, 2002; Roth and Dicke, 2005; Schoenemann et al., 2005).

\footnotetext{
Received June 25, 2012; revised Jan. 24, 2013; accepted Jan. 31, 2013

Author contributions: W.D.H. designed research; W.D.H. and B.A. performed research; W.D.H. and B.A. analyzed data; W.D.H. and B.A. wrote the paper.

Acknowledgement: This research was supported by NIH Grants NS-42867, NS-73134, HD-56232, and HD-60563 to W.D.H. and MH-80892 and EB06266 to B.V. We thank Yerkes National Primate Research Center veterinary staff for assistance in MR imaging. American Psychological Association guidelines for the treatment of animals were followed during all aspects of this study.

The authors declare no competing financial interests.

Correspondence should be addressed to William D. Hopkins, Neuroscience Institute, Georgia State University, P.0. Box 5030, Atlanta, GA 30302. E-mail: whopkins4@gsu.edu.

DOI:10.1523/JNEUROSCI.2996-12.2013

Copyright $\odot 2013$ the authors $\quad 0270-6474 / 13 / 335241-08 \$ 15.00 / 0$
}

An important aspect of cortical organization that has not been examined from a comparative primate perspective is cortical thickness. Studies of postmortem human brains have shown that there are significant regional and lateralized differences in cortical thickness. For instance, in postmortem material, cortical thickness varies from $\sim 2-4 \mathrm{~mm}$ across the human cerebrum, with thinner cortex found in primary motor and sensory regions compared with thicker cortex in association cortex (Rabinowicz et al., 1999). Recent advances in in vivo imaging technologies used with human brains have largely validated these findings (Luders et al., 2006b), when derived from T1-weighted structural magnetic resonance imaging (MRI) scans (Luders et al., 2006b). In terms of lateralization, small but significant asymmetries in cortical thickness have been found in the human brain including leftward biases for the precentral gyrus (PreCG), middle frontal, anterior temporal, and superior parietal lobes (SPLs). Significant rightward biases have been reported for inferior posterior temporal lobe and inferior frontal gyrus (IFG) (Luders et al., 2006a) with some evidence that these effects are influenced by gender (Im et al., 2006).

Cortical thickness is relatively conserved in primate brain organization (Changizi, 2001; Sherwood and Hof, 2007; Gorrie et al., 2008). Whether thickness varies in relation to different cortical regions and lateralization in closely related primates, such as chimpanzees, is virtually unknown but may provide important information on primate brain evolution and the mechanisms that underlie human cognitive and motor specializations. The aim of this study was to quantify regional and lateralized variation in cortical thickness in chimpanzees. In addition, we also computed the surface area and cortical gray and white matter volumes. Seldon (2005) and others (Giedd et al., 1999; Sowell et 
al., 2004; Shaw et al., 2006) have reported that there is an inverse association between white matter volume and cortical thickness during human development. More recently, in humans from different age groups, Hogstrom et al. (2012) has reported that there is a positive association between white matter volume and cortical surface and significant negative associations between these two measures and cortical thickness. Seldon (2005) has hypothesized that, during development, white matter grows outward and expands the outer surface of the cortex much like a balloon. The consequence of this expansion is that outer gray matter (GM) surface has to thin out to cover the expanding surface area. Based on the theory proposed by Seldon (2005), we hypothesized that, like humans, chimpanzee subjects with either larger white matter volumes or cortical surface areas would have thinner cortical thickness values.

\section{Materials and Methods}

Subjects

MRIs were obtained in 71 captive chimpanzees ( 23 males, 48 females). The subjects ranged in age from 11 to 55 years (mean, 22.34; SD, 11.11) and were all housed at the Yerkes National Primate Research Center. To evaluate the potential effect of handedness on asymmetries in cortical thickness, we also compared right, left, and ambiguously handed individuals in the analyses of AQ scores. Handedness was measured on a task that requires coordinated bimanual actions, referred to as the tube task. Binomial $z$-scores based on the frequency of right- and left-hand use were used to classify the hand preference of the chimpanzees. Apes with $z$-scores $>1.95$ or -1.95 were classified as right and left handed. Chimpanzees with $z$-scores $>-1.96$ or $<1.96$ were classified as ambiguously handed (i.e., did not have a significant hand preference) Within the sample, there were 24 left-, 13 ambiguously, and 34 right-handed chimpanzees All scanning procedures were approved by the University Institutional Animal Care and Use committee and followed the guidelines outlined by the Institute of Medicine.

\section{Magnetic resonance image collection}

All chimpanzees were scanned during one of their yearly physical examinations. MRI scans followed standard procedures at the Yerkes National Primate Research Center. Subjects were first immobilized with ketamine $(10 \mathrm{mg} / \mathrm{kg})$ or telazol $(3-5 \mathrm{mg} / \mathrm{kg})$ and subsequently anesthetized with propofol $[40-60 \mathrm{mg} /(\mathrm{kg} / \mathrm{h})]$. Each subject was then transported to the MRI scanner and placed in a supine position in the scanner with their head in a human-head coil. Upon completion of the MRI, chimpanzees were temporarily singly housed for $2-12 \mathrm{~h}$ to permit close monitoring and safe recovery from the anesthesia before return to the home social group. Chimpanzees were scanned using a 3.0 tesla scanner (Siemens Trio, Siemens Medical Solutions USA). T1-weighted images were collected using a three-dimensional gradient echo sequence (pulse repetition $=2300 \mathrm{~ms}$, echo time $=4.4 \mathrm{~ms}$, number of signals averaged $=3$, matrix size $=320 \times 320$, with $0.6 \times 0.6 \times 0.6$ resolution).

\section{Image processing and cortical thickness measurement}

The MRI scans were processed in a pipeline that required several steps including skull stripping using FSL (Smith et al., 2004), diffeomorphic registration to a symmetric population-specific template chimpanzee brain (Avants et al., 2011b), and subsequent probabilistic segmentation of the T1-weighted images into gray matter, white matter, and CSF following procedures and software that have been described in detail previously (Avants et al., 2008, 2010a).

After brain extraction, we used the openly available Advanced Normalization Tools (ANTS; http://www.picsl.upenn.edu/ANTS/) to perform multivariate normalization and structure-specific processing of our data (Avants et al., 2008; Klein et al., 2009). ANTS encodes current best practices in image registration, optimal template construction, and segmentation and is scalable to large-scale, distributed computing environments. ANTS cross-sectional studies deform each individual dataset into a standard local template space and/or a canonical stereotactic coordinate system. The core processing maps T1 structural MRI to an optimal
Table 1. Mean cortical thickness ( \pm SE) for each hemisphere and region

\begin{tabular}{lllllrl}
\hline & Left & SE & Right & SE & $t$ & $p$ \\
\hline Frontal lobe & & & & & & \\
$\quad$ Dorsal PFC & 2.67 & 0.03 & 2.71 & 0.03 & 3.801 & 0.000 \\
$\quad$ Orbital PFC & 2.56 & 0.03 & 2.53 & 0.04 & 3.269 & 0.001 \\
Mesial PFC & 2.43 & 0.04 & 2.46 & 0.03 & 1.213 & 0.229 \\
IFG & 2.78 & 0.05 & 2.78 & 0.05 & 3.151 & 0.002 \\
$\quad$ Precentral & 1.67 & 0.04 & 1.75 & 0.04 & 6.643 & 0.000 \\
Parietal & & & & & & \\
$\quad$ Postcentral & 1.62 & 0.04 & 1.71 & 0.04 & 5.163 & 0.000 \\
$\quad$ Superior & 2.76 & 0.06 & 2.83 & 0.06 & 3.499 & 0.001 \\
$\quad$ Supramarginal & 2.45 & 0.04 & 2.51 & 0.05 & 3.807 & 0.000 \\
Temporal & & & & & & \\
$\quad$ Superior & 2.16 & 0.05 & 2.23 & 0.05 & 4.820 & 0.000 \\
$\quad$ Middle & 2.70 & 0.04 & 2.83 & 0.04 & 8.131 & 0.000 \\
$\quad$ Inferior & 2.65 & 0.04 & 2.61 & 0.05 & -1.425 & 0.158 \\
$\quad$ pSTG & 2.08 & 0.06 & 2.18 & 0.06 & 3.396 & 0.001 \\
\hline
\end{tabular}

Analyses of interhemispheric differences in grey matter thickness were performed using pared samples $t$ tests. Values indicate grey matter thickness in millimeters.

template space, which is defined as the population-specific, unbiased average shape and appearance image derived from a representative population (Avants and Gee, 2004; Avants et al., 2010b). The average template was constructed to optimally represent both the original and a flipped version of the dataset, such that the final template is symmetric about the midsagittal plane. The coordinate deformations themselves are smooth and invertible, that is, diffeomorphic - neuroanatomical neighbors remain neighbors under the mapping. At the same time, the algorithms used to create these deformations are biased toward the reference space chosen to compute the mappings. Moreover, these topologypreserving maps capture the large deformation necessary to aggregate populations of images in a common space. Recent evaluation studies suggest that ANTS-based normalization is currently the most stable and reliable method available. After defining the template image to target image coordinate transformation, we use template-based priors and $\mathrm{N} 4$ inhomogeneity-filed correction to accurately segment cortical gray matter segmentation and perform cortical parcellation (Das et al., 2009). After these initial processing steps, the diffeomorphic registration-based cortical thickness (DiReCT) method is used to compute cortical thickness (Das et al., 2009).

DiReCT uses the segmentation probability images to compute a continuous voxelwise estimate of cortical thickness (Das et al., 2009). Di$\mathrm{ReCT}$ is unique in that it exploits tissue segmentation probability maps to identify a maximum likelihood correspondence between the white matter surface and the outer gray matter surface where the correspondence mapping is constrained to be spatially regular, differentiable, and invertible, i.e., diffeomorphic. As a consequence, DiReCT thickness estimates incorporate both shape constraints and subtle probabilistic information about the likely position of sulci that may not be visible in a hard segmentation. Thus, DiReCT is a robust image-based technique for identifying voxelwise and regional thickness information. An independent implementation and validation of this method showed that it is competitive with Freesurfer, a commonly used program for estimating cortical thickness (Clarkson et al., 2011). We note, however, that the implementation used by Clarkson et al. (2011) was never itself directly compared with that available in the ANTS toolkit and we suspect that differences between the Clarkson et al. implementation and our own gold standard implementation may exist.

As has been done in some studies of cortical thickness in humans and monkeys (Styner et al., 2007; Van Essen et al., 2012), we used a seed or region-of-interest approach to quantify GM thickness in 12 select areas of interest (Table 1), including the (1) dorsal, mesial, and orbital prefrontal cortex; (2) superior, middle, and inferior temporal gyri; (3) precentral and postcentral gyri; (4) inferior frontal gyrus; (5) posterior superior temporal gyrus (pSTG); (6) supramarginal gyrus (SMG); and (7) superior parietal lobe. The landmarks used to define each region of interest are provided below and they were selected for theoretical and pragmatic reasons. Specifically, there has been considerable comparative interest in 


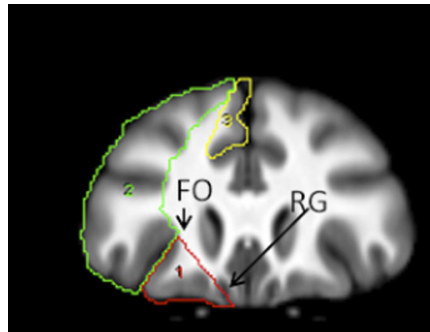

a.

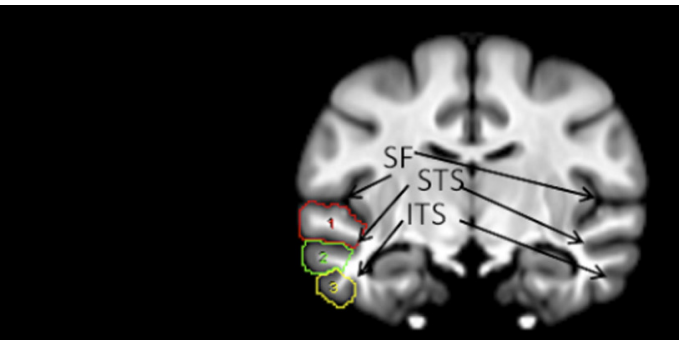

b.

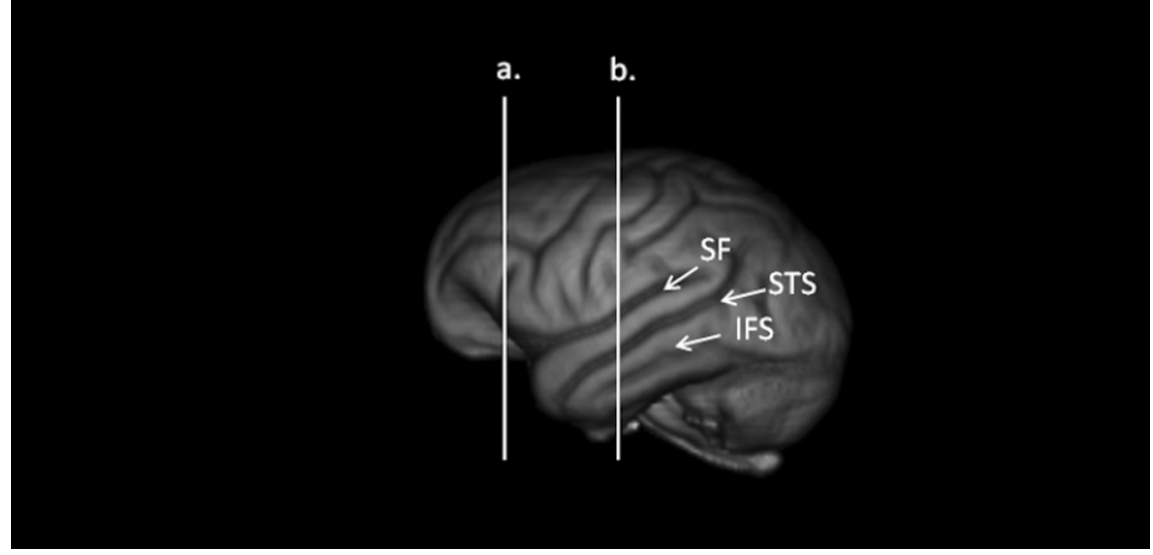

Figure 1. Bottom, 3D-reconstruction of chimpanzee template brain with vertical white lines representing regions outlined above $(\boldsymbol{a}, \boldsymbol{b})$. $\boldsymbol{a}$, Coronal view of the chimpanzee brain with the orbital (red), dorsal (green), and mesial (yellow) prefrontal cortex regions outlined on the scan. $\mathrm{RG}$, Rectus. $\boldsymbol{b}$, Coronal view of the chimpanzee brain with the superior (red), middle (green) and inferior (yellow) temporal gyri regions outlined on the scan.

the evolution of the prefrontal, temporal, and parietal cortex (Deacon, 1997; Schoenemann et al., 2005; Rilling, 2006; Sherwood et al., 2012), and, therefore, we focused on these regions. Similarly, there has been significant interest in the evolution of cortical organization and lateralization in the homologs to Broca's and Wernicke's area in chimpanzees (Cantalupo and Hopkins, 2001; Keller et al., 2009; Hopkins and Nir, 2010; Schenker et al., 2010; Spocter et al., 2010), and this was our reasoning for quantifying the inferior frontal gyrus and posterior superior temporal gyri. Finally, we also included the precentral and postcentral gyri as regions of interest because they are primary motor and sensory cortices and we hypothesized that cortical thickness would be lower in these regions compared with the others. The region of interest masks were drawn on the chimpanzee template brain using a mouse-controlled pointer and then transformed back to the individual GM thickness maps for each subject, using the inverse matrix of the original registration. The masks were then applied to the individual subjects' GM thickness map to derive average thickness measures for each of the 12 regions within each hemisphere. We also computed an average cortical thickness measure for each hemisphere. This was done by drawing a mask that covered the entire left or right hemisphere, excluding the brainstem and cerebellum. We note that, because the template is symmetric, the masks only had to be drawn on one hemisphere of the template brain and could then be transformed to label both left and right sides of the individual subject's brain.

\section{Regions of interest}

PFC. We adopted the landmarks used by Semendeferi et al. (1997) in defining the orbital, dorsal, and mesial prefrontal cortex in serial coronal images (Fig. 1). Beginning posteriorly when the frontal orbital (FO) sulcus was first visible, for the orbital PFC, a line was drawn from the surface of the FO sulcus to its most medial point. A second line was then drawn from the medial point of FO to the tip of the rectus gyrus, which was then followed along the inferior outer surface of the cortex until intersecting with the lateral point of the FO sulcus. If the caudate and putamen were visible on the images, the line extending from the medial FO sulcus to the rectus gyrus excluded these regions. The dorsal PFC was outlined by laterally tracing on the surface of the brain from FO to the upper, dorsal-most mesial point. From there, a line was drawn that connected the most medial point of all frontal sulci between the most mesial point and the FO sulcus. Finally, the mesial PFC was defined from the gray matter tip of the rectus gyrus to the upper dorsal end of the mesial surface, which included portions of the superior frontal gyrus but omitted any tissue belonging to the anterior cingulate cortex and subgenu region.

Temporal lobe cortex. We adopted similar landmarks to those used by Rilling and Seligman (2002) and quantified the superior (STG), middle (MTG), and inferior temporal gyri (ITG) (Fig. 1). These three regions were primarily traced in the coronal plane. The superior and inferior borders of the STG were the sylvian fissure (SF) and superior temporal sulcus (STS). For all images in which the SF and STS were clearly visible, the entire gyrus was outlined with a mouse-driven cursor from the most medial point of the SF, laterally to the surface of the brain and then to the most medial point of the STS. The superior and inferior borders of the MTG were the STS and inferior temporal sulcus (ITS). Like the STG, for all images in which the STS and ITS were clearly visible, the entire gyrus was outlined with a mouse-driven cursor from the most medial point of the STS, laterally to the surface of the brain, and then to the most medial point of the ITS. The superior and inferior borders for the ITG were the ITS and the lateral occipital temporal sulcus. The entire gyrus between the medial border of the ITS to the lateral surface, then to the most medial point of the lateral occipital temporal sulcus, were traced on images in which both sulci were visible.

SPL. In the transverse plane, the lateral border was the intraparietal sulcus (IPS) and the medial border was the superior parietal sulcus (SPS) (Fig. 2). On serial transverse images in which the IPS and SPS could be identified, the gyrus between these two sulci was traced. On each image, the IPS sulcus was traced from its most anterior to posterior point. A line was then drawn between the most posterior points of the IPS and SPS. We then followed the SPS from its most posterior to most anterior point. Last, a line was drawn between the two most anterior points of the IPS and SPS.

$S M G$. This region was primarily traced in the transverse plane and included the entire gyrus between the IPS, the superior postcentral sulcus (SPCS), and the lateral surface of the cortex (Fig. 2). The inferior border of the SMG was the point at which the IPS connected to the SPCS. Moving dorsally, the SMG was traced on all slices in which the SPCS and IPS were visible. On each image, a line was drawn starting at the most lateral edge of SPCS to the most posterior point of the IPS. Next, a line was traced along the lateral surface of the cortex between the most posterior point of IPS and the lateral point of the SPCS. Tracing continued on serial images until the IPS was no longer visible.

PreCG. This region was traced in the sagittal and transverse planes. The PreCG was the entire gyrus between the central sulcus (CS) and the precentral sulcus, including the inferior $(\mathrm{PCI})$ and superior $(\mathrm{SPC})$ portions (Fig. 2). The CS was the posterior border of the PreCG. Ventrally, we used PCI sulcus as the anterior border. Moving dorsally, PCI terminates and at that point we used the SPC sulcus as the anterior border of the PreCG. On each image, we drew a line from the most medial point of PCI to the lateral edge of the cortex. We then drew a line on the lateral edge of the cortex between PCI and CS, and then drew a line to the most medial point of the CS.

Postcentral gyrus. The anterior border of the postcentral gyrus (PostCG) was the CS and the posterior border was the postcentral sulcus (PCS) (Fig. 2). For all images in the transverse plan in which the CS and 
IPCS could be seen, the entire gyrus was traced. On each image, a line was drawn from the most medial point of CS to the outer cortex. We then drew a line along the outer cortex from the lateral tip of CS to PCS, then traced along PCS to its most medial point.

IFG. The anterior border of the IFG in chimpanzees is the FO, the posterior border is the $\mathrm{PCI}$, and the superior border is the inferior frontal sulcus (IFS) (Fig. 3). Following methods previously used by Hopkins et al. (2008), we created a mask by tracing the entire gyrus between PCI and FO on serial dorsal to ventral images in the transverse plane until PCI terminated. On each image, we drew a line from the most medial point of PCI to the lateral surface of the cortex. We then drew a line along the surface of the cortex between PCI and FO and then drew a line to the most lateral point of FO. Finally, a line was drawn between the medial points of the FO and PCI sulci.

pSTG. The pSTG was quantified using methods and landmarks that have been used previously in human and nonhuman primate brains (Cantalupo et al., 2003; Lyn et al., 2011). The anterior border of the PSTG was the first slice in the coronal plane in which the inferior branch of insular sulcus terminated. From that point, as described above, we traced the entire gyrus using SF as the superior border and the inferior border was the superior temporal sulcus (STS). The entire gyrus between the SF and STS were traced on all slices until the SF terminated posteriorly (Fig. 3).

\section{Surface area, gray, and white} matter volume

We use a classic expectation maximization framework (Atropos, available within ANTS) and N4 bias field correction (available in ITK and ANTS) to find a maximum a posteriori brain segmentation guided by probabilistic tissue priors (Avants et al., 2011a). The tissue priors provide both initialization and spatial guidance to the tissue classification, and the same segmentation maps were used to estimate white matter volume, gray matter volume, and cortical thickness. Object masks were drawn over the entire left and right hemispheres for each subject and subsequently applied to the segmented GM and WM volumes to calculate volumes within each half of the brain. The cerebellum and related brainstem structures were excluded from calculations in total gray and white matter volume. To compute the surface area, we used BrainVisa (BV), an automated program that determines the outer cortical folding of the left and right cerebral cortex excluding the cerebellum and brainstem.

\section{Data analysis}

Average thickness measures for each region were obtained by summing the thickness measures for left and right hemispheres and dividing by two. Asymmetry quotients (AQ) for each region were derived following the formula $[\mathrm{AQ}=(\mathrm{R}-\mathrm{L}) / \mathrm{R}+\mathrm{L})$ where $\mathrm{R}$ and $\mathrm{L}$ indicate the cortical thickness for the left and right region. Unless otherwise noted, parametric statistics were used for all analyses with $\alpha$ set to $p<0.05$. Post hoc tests, when necessary, were conducted using Tukey's Honestly Significant difference $(p<0.05)$.

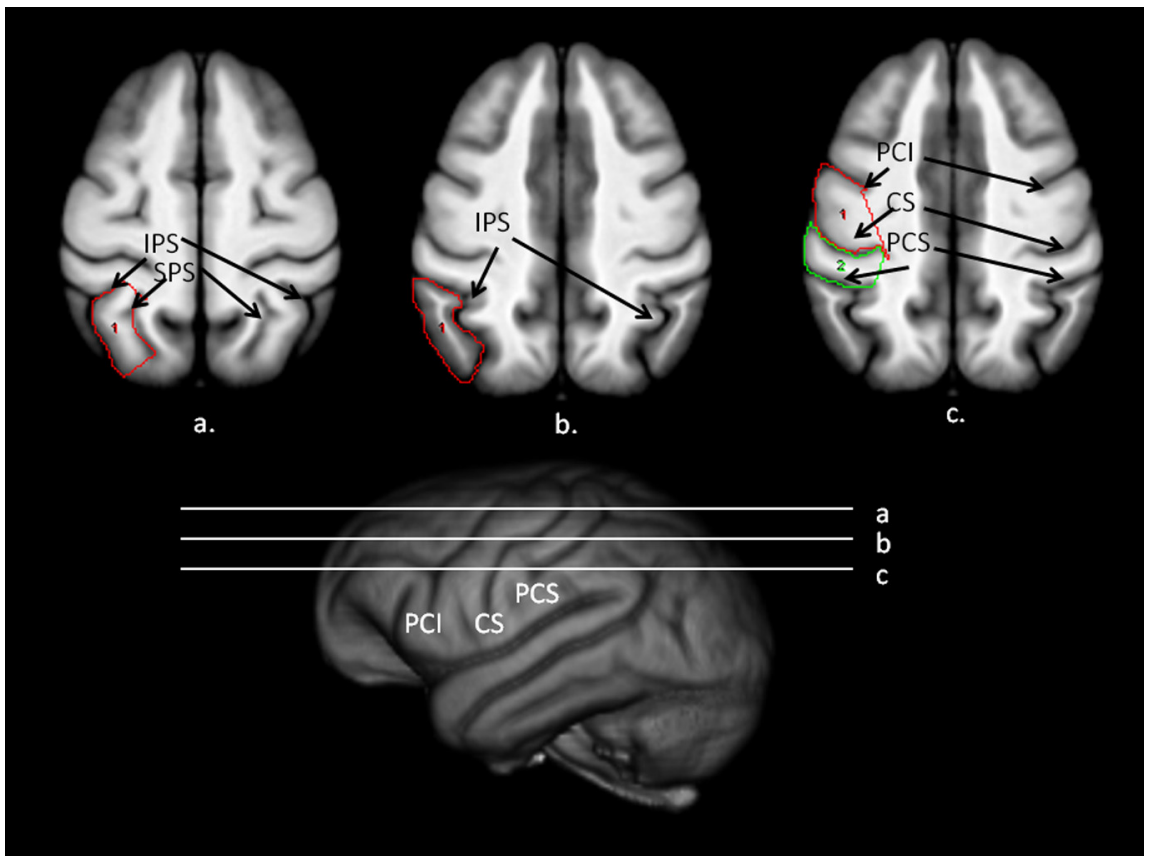

Figure 2. Bottom, 3D-reconstruction of chimpanzee template brain with horizontal white lines representing regions outlined above $(\boldsymbol{a}-\boldsymbol{c}) . \boldsymbol{a}$, Transverse view of the chimpanzee brain with superior parietal lobe. $\boldsymbol{b}, \boldsymbol{c}$, Supramarginal gyrus $(\boldsymbol{b})$ and precentral $(c$, red) and postcentral (green) outlined on the scan.

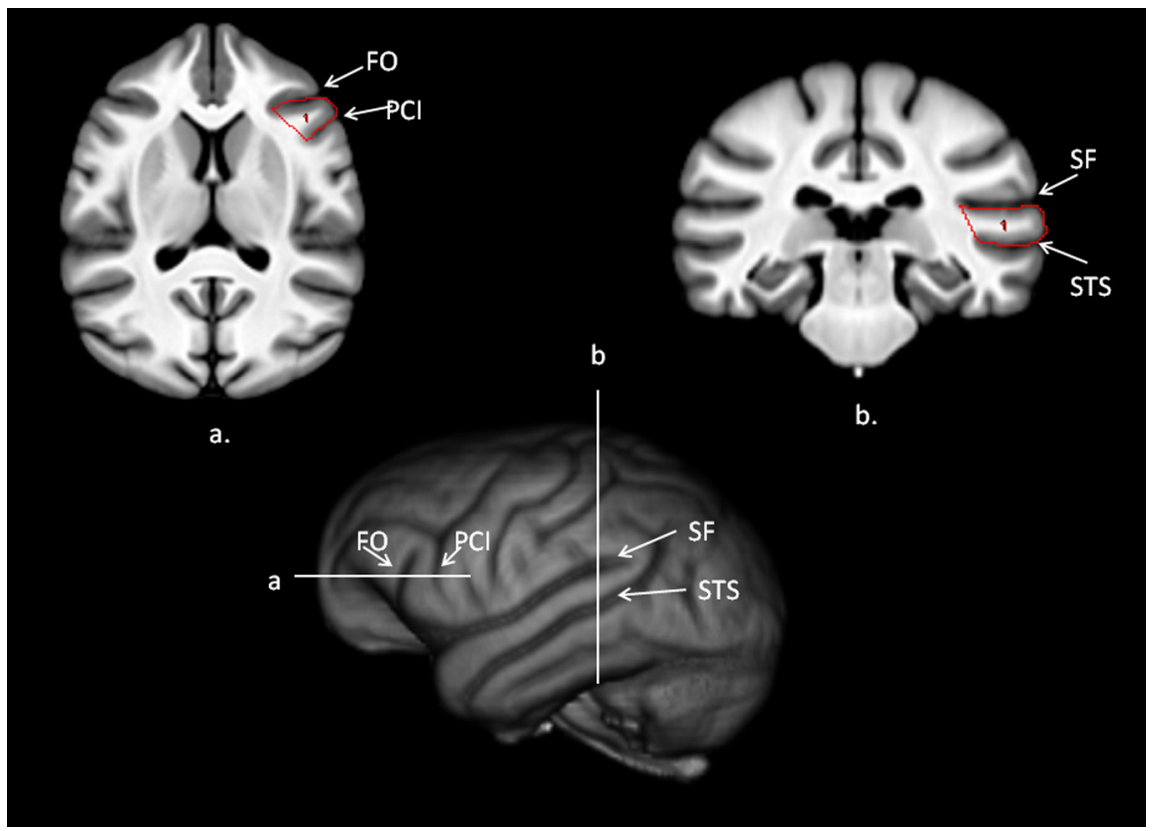

Figure 3. Bottom, 3D-reconstruction of chimpanzee template brain with horizontal and vertical white lines representing regions outlined above $(\boldsymbol{a}, \boldsymbol{b}) . \boldsymbol{a}$, Transverse view of the chimpanzee template brain with the IFG outlined on the scan. $\boldsymbol{b}$, Coronal view of the chimpanzee template brain with the posterior superior temporal gyrus outlined on the scan.

\section{Results}

Global and regional variation in cortical thickness

We initially tested for regional variation in cortical thickness using a mixed model ANOVA. Region was the repeated measure while sex was the between group factor. The chimpanzees showed significant variation in gray matter thickness throughout the cortex $F_{(11,759)}=$ 144.71, $p<0.001$ (Fig. 4; Table 1) (Fig. 5). Post hoc analysis indicated that, as is the case in human brain, cortical thickness was lower in primary sensory and motor regions including the precentral and 


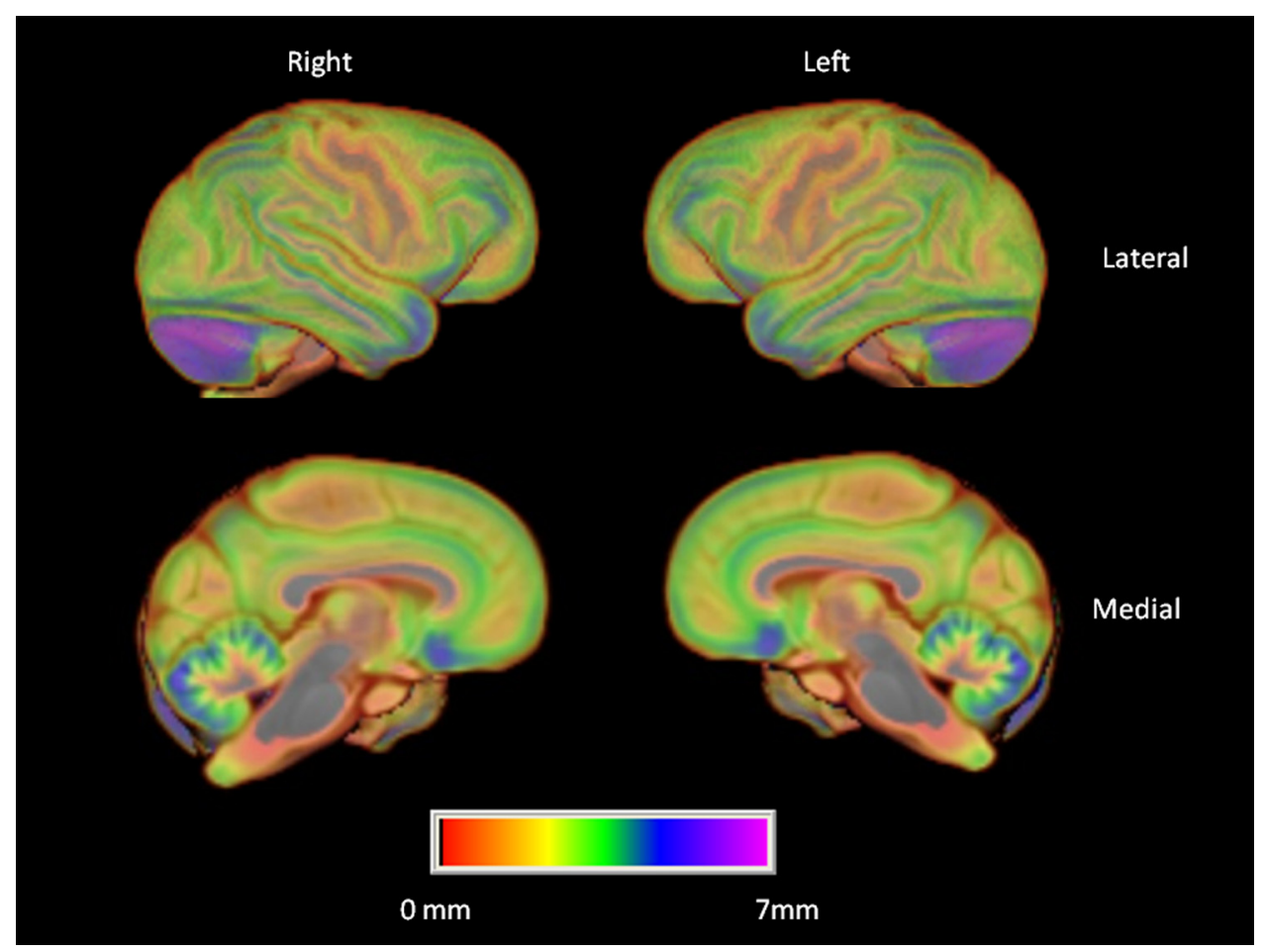

Figure 4. Mean cortical thickness of the left and right hemispheres. Color bar indicates variation in cortical thickness throughout cortex. Top, Left and right lateral view. Bottom, Left and right medial views.

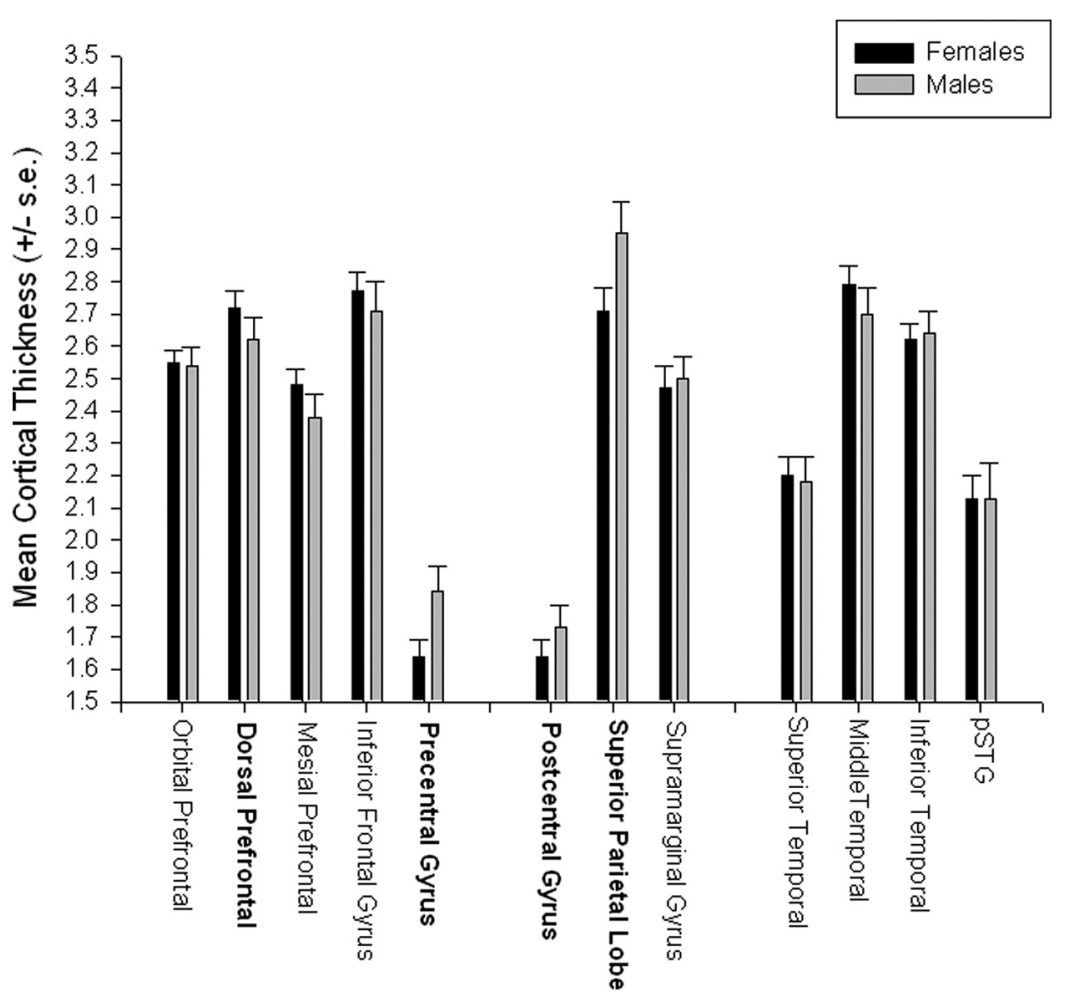

Figure 5. Mean cortical thickness ( \pm SE) for male and female chimpanzees for each of the 12 regions. Bolded labels indicate significant male-female differences at $p<0.05$ after adjustment for overall brain volume.

postcentral gyri compared with all other cortical regions (Fig. 4). In addition, a significant two-way interaction was found between sex and region $F_{(11,759)}=2.28, p<0.009$ (Fig. 4). Subsequent post hoc analysis indicated no significant differences between males and females for any of the regions.

\section{Adjusted thickness measures}

Luders et al. (2006b) found that regional and sex differences in cortical thickness can be influenced when adjustments are made for variation in brain volume. To address this specific issue, we conducted a second analysis examining sex and regional variation after adjusting for total gray and white matter volume. For this analysis, we reran the mixed-model ANOVA using the thickness values as the repeated measure while sex was the between group factor, but we included brain volume as a covariate. As before, we found a significant main effect for region $F_{(11,759)}=242.18, p<0.001$ and a significant two-way interaction between sex and region $F_{(11,759)}=3.57, p<0.001$. Males were found to have thicker cortex than females for the precentral and postcentral gyri as well as the superior parietal lobe. In contrast, females were found to have thicker dorsal PFC than males.

\section{Lateralization in cortical thickness}

We next considered asymmetries in each of the regions. After adjusting $\alpha$ for multiple comparisons, one sample $t$ tests on the AQ scores revealed significant rightward asymmetries in cortical thickness for 10 of the 12 regions including orbital and dorsal prefrontal cortex, precentral and postcentral gyri, superior parietal lobe, supramarginal gyrus, superior temporal gyrus, middle temporal gyrus, inferior frontal gyrus, and posterior superior temporal gyrus (Table 1). The rightward asymmetries in 
Table 2. Mean surface area, grey and white matter volumes for the left and right hemispheres in male and females chimpanzees

\begin{tabular}{llll}
\hline & Males & Females & Total \\
\hline $\begin{array}{llll}\text { Grey matter } \\
\text { LH }\end{array}$ & $93.73(1.94)$ & $83.50(1.35)$ & \\
$\quad$ RH & $99.34(0.178)$ & $89.42(1.24)$ & $94.62(1.18)$ \\
White matter & & & \\
LH & $73.15(1.56)$ & $72.14(1.07)$ & $72.65(1.37)$ \\
RH & $72.67(1.55)$ & $70.64(1.07)$ & $71.65(1.31)$ \\
Surface area & & & \\
LH & $187.96(2.45)$ & $178.05(1.69)$ & $183.00(1.49)$ \\
RH & $187.43(2.45)$ & $176.52(1.74)$ & $181.98(1.53)$ \\
\hline
\end{tabular}

Surface area, grey and white matter volumes are in cubic centimeters. Numbers in the parentheses are SEs.

gray matter thickness based on the region of interest approach were largely confirmed when using a voxel-based approach (see http://stnava.github.com/WHopkinsNHP/).

Because population-level asymmetries in cortical thickness were found for 10 regions and most regions showed some level of skewness, we opted to use non-parametric Mann-Whitney U tests to assess sex differences in the AQ scores. Sex differences were found for 2 regions including the inferior frontal gyrus $(z=$ $-2.04, p<0.05)$ and precentral gyrus $(z=-2.73, p<0.007)$. Females showed greater rightward asymmetries than males for both the inferior frontal gyrus and precentral gyrus. All of the sex differences in AQ scores were small and, after correcting $\alpha$ for multiple comparisons $(p<0.004)$, none of the differences remained significant, so the results should be interpreted with some caution. Kruskal-Wallis tests comparing the AQ scores for each region between left-, ambiguously, and right-handed chimpanzees failed to reveal any significant differences.

\section{Surface area, gray, and white matter volume}

For the surface area (SA), GM, and WM volumes, three repeatedmeasure ANOVAs were performed with hemisphere (left, right) as the repeated measure and sex as the between group factor. The mean SA, GM, and WM volumes for the left and right hemisphere in males and females are shown in Table 2. For gray matter, significant main effects for $\operatorname{sex} F_{(1,69)}=21.71, p<0.001$ and hemisphere $F_{(1,69)}=92.52, p<0.001$ were found. Males had significantly higher gray matter volumes than females and a significant rightward asymmetry was found. No other significant main effects or interactions were found. For white matter, a significant main effect for hemisphere was found $F_{(1,69)}=14.49, p<$ 0.001 . White matter volume was larger in the left compared with right hemisphere. No other significant main effects or interactions were found. Finally, for SA, a significant sex difference was found $F_{(1,69)}=13.01, p<0.001$ with males having a larger surface area then females.

\section{Correlations between cortical thickness, surface, gray, and white matter volume}

In this analysis, we correlated the average GM, WM, and SA volumes with the average cortical thickness for each region of interest (Table 3). In this initial analysis, we used the average cortical thickness for the entire cortex instead of the thickness measures for each region to minimize the number of analyses and reduce the likelihood of type I error. The results of these analyses are shown in Table 3. As can be seen, significant negative associations were found between average cortical thickness and GM and WM volumes but not surface area. Chimpanzees with thinner cortex had larger GM and, particularly, WM vol-
Table 3. Intercorrelations between volume and asymmetry values for SA, GM, WM, and cortical thickness

\begin{tabular}{|c|c|c|c|c|}
\hline & WM & GM & SA & Thickness \\
\hline \multicolumn{5}{|l|}{ Volume } \\
\hline WM & 1.0 & & & \\
\hline GM & $0.457^{* * *}$ & 1.0 & & \\
\hline SA & 0.254 & 0.585 & 1.0 & \\
\hline Thickness & $-0.506^{* * *}$ & $-0.263^{*}$ & -0.148 & 1.0 \\
\hline \multicolumn{5}{|l|}{ Asymmetry } \\
\hline WM & 1.0 & & & \\
\hline GM & 0.045 & 1.0 & & \\
\hline SA & $0.241^{*}$ & 0.056 & 1.0 & \\
\hline Thickness & $-0.529^{* * *}$ & 0.195 & -0.054 & 1.0 \\
\hline
\end{tabular}

umes. We also found significant positive associations between cortical surface area, GM and WM volumes.

We also performed correlation analyses between the AQ measures for GM, WM, SA, and average thickness and these are also shown in Table 3. A significant positive association was found between SA and WM AQ scores. Subjects with greater rightward asymmetries in WM had similarly greater rightward asymmetries in SA. Significant negative correlations were found between the AQ scores for WM volume and average cortical thickness. Subjects with greater rightward asymmetries in cortical thickness had greater leftward asymmetries in WM volume.

\section{Discussion}

The results reported here are the first evidence of regional variation and lateralization in cortical thickness in chimpanzees. There were three significant findings. First, as is the case with humans, chimpanzees show regional variation in cortical thickness with thinner cortex in primary motor and sensory cortex and thicker gray matter in association cortex including prefrontal cortex and portions of the parietal and temporal lobes. We also found some evidence of regional sex differences in cortical thickness. Second, our findings indicate that chimpanzees show robust population-level rightward asymmetries in gray matter thickness and a concomitant leftward asymmetry in white but not gray matter volume. Third, white but not gray matter volume was negatively correlated with cortical thickness. Thus, subjects with greater white matter volume had thinner gray matter thickness.

After adjustment for overall brain size, we found four significant regional sex differences in cortical thickness in the chimpanzees including the precentral and postcentral gyri superior parietal lobe and dorsal prefrontal cortex. For all three of the four regions, males had higher cortical thickness measures than the females. It should also be noted that males were found to have significantly larger gray but not white matter volumes (Table 2). We had more than twice as many females than males in the sample and the age ranges were quite variable within our chimpanzee sample. However, when we matched all 23 males with females of the same age and reran the analyses, all the significant sex differences found in the original analysis remained significant. Thus, the disparities in sample sizes between sexes and the within sex variability in age does not seem to explain the results. In short, the differences appear to be relatively small but not due to other factors such as age.

Sex differences in gray matter thickness have also been reported in humans and they appear to somewhat differ from those reported here in chimpanzees. Luders et al. (2006b) reported that females had thicker cortex bilaterally in the precentral and postcentral regions, the occipital lobe, and the anterior tip of the 
temporal lobe. Males did not differ from females in cortical thickness for any region and this was the case when these authors scaled or did not scale the thickness measures on the basis of differences in brain size. Im et al. (2006) also reported sex difference in cortical thickness in humans and their results were not all that different from the findings reported by Luders et al. (2006). Specifically, Im et al. (2006) found that females were found to have thicker cortex than males, particularly in the frontal, parietal, and occipital regions. Moreover, there was not a single brain region that was thicker in males compared with females. When the findings of these two studies are compared with those reported here in chimpanzees, it suggests that sex differences in gray matter thickness may differ between humans and chimpanzees. The functional and evolutionary significance of these differences are not clear but the results seem to be evident even considering some potential confounds within our sample as well as between sexes.

The chimpanzees showed prominent rightward asymmetries in cortical thickness and significant leftward asymmetries in white matter volume. Chimpanzees with greater rightward asymmetries in cortical thickness were also found to have greater leftward asymmetries in white matter. Furthermore, without consideration of asymmetries, chimpanzees with larger white matter volumes were found to have thinner overall cortical thickness (Table 3). When considered together, the findings complement each other and suggest that that the rightward cortical thickness asymmetries may be attributable to the expanded white matter volumes in the left compared with right hemisphere. Significant population-level white matter asymmetries have previously been found in the perisylvian regions of the chimpanzees brain (Cantalupo et al., 2009) as well as with voxel-based morphometry (Hopkins et al., 2010). More recently, using diffusion tensor imaging, leftward asymmetries have been found in arcuate fasciculus of chimpanzees, the major white matter tract connecting the superior temporal gyrus with the inferior frontal gyrus (Rilling et al., 2011). Thus, multiple methods have revealed leftward asymmetries in white matter volume, spatial location ( $\mathrm{Li}$ et al., 2010), and connectivity in chimpanzees, and this is likely linked to regional variation in lateralization in cortical thickness.

Alternatively, the rightward asymmetries found in cortical thickness may be attributable to a larger left hemisphere surface area, including within the cortical folds. Significant populationlevel leftward asymmetries have been found in the surface areas and depths of several cortical sulci in chimpanzees, including the sylvian fissure, fronto-orbital, and superior precentral, intraparietal, and postcentral inferior and lunate sulci (Bogart et al., 2012). Recall that rightward population-level asymmetries were found in gray matter volume. Thus, in this explanation, the volume of gray matter in the left compared with right hemisphere has to be expanded over a larger surface area, which results in increased cortical thinning. It should be noted that we did not find a significant association between surface area and cortical thickness, and therefore this interpretation was not directly supported by the findings reported here. Nonetheless as has been reported in humans, cortical surface area was positively correlated with white matter volume in the chimpanzees (Table 3). Thus, it is likely that both surface area and white matter volume have some influence on cortical thickness, although our results suggest that white matter plays a more significant role.

The underlying mechanisms that determine population-level asymmetries in humans and other primates remain unclear but the comparative data continue to challenge the long held belief that they are unique to humans and explicitly linked to the evo- lution of language and speech, as suggested by some (Corballis, 1992; McGrew and Marchant, 1997; Crow, 2010). We would suggest that anatomical and functional asymmetries were present in the common ancestor of humans and chimpanzees and that each species developed their own patterns of asymmetry that reflect their unique ecological adaptations rather than the occurrence of a saltational event that resulted in the emergence of asymmetries after the split between chimpanzees and humans.

In summary, this is the first report of regional and lateralized variation in cortical thickness in chimpanzees. Like human brains, chimpanzees show thinner cortex in the primary motor and somatosensory cortex compared with association cortex. Moreover, chimpanzees show small but significant populationlevel asymmetries in cortical thickness, as has been reported in human brains. The functional significance of individual differences in cortical thickness in chimpanzees remain unclear but future studies should focus on establishing whether variability in cognitive performance or other behavioral phenotypes are linked to cortical thickness (Gorrie et al., 2008).

\section{Notes}

Supplemental material for this article is available at http://stnava.github. com/WHopkinsNHP/. This material has not been peer reviewed.

\section{References}

Avants B, Gee JC (2004) Geodesic estimation for large deformation anatomical shape and intensity averaging. Neuroimage 23 [Suppl 1]: S139-S150. Medline

Avants BB, Epstein CL, Grossman M, Gee JC (2008) Symmetric diffeomorphic image registration with cross-correlation: evaluating automated labeling of elderly and neurodegenerative brain. Med Image Anal 12:26-41. Medline

Avants BB, Cook PA, Ungar L, Gee JC, Grossman M (2010a) Dementia induces correlated reductions in white matter integrity and cortical thickness: a multivariate neuroimaging study with sparse canonical correlation analysis. Neuroimage 50:1004-1016. CrossRef Medline

Avants BB, Yushkevich P, Pluta J, Minkoff D, Korczykowski M, Detre J, Gee JC (2010b) The optimal template effect in hippocampus studies of diseased populations. Neuroimage 49:2457-2466. CrossRef Medline

Avants BB, Tustison NJ, Wu J, Cook PA, Gee JC (2011a) An open source multivariate framework for $\mathrm{n}$-tissue segmentation with evaluation on public data. Neuroinformatics 9:381-400. CrossRef Medline

Avants BB, Tustison NJ, Song G, Cook PA, Kelin A, Gee JC (2011b) A reproducible evaluation of ANTS similarity metric performance in brain image registration. Neuroimage 54:2033-2044. CrossRef

Bogart SL, Mangin JF, Schapiro SJ, Reamer L, Bennett AJ, Pierre PJ, Hopkins WD (2012) Cortical sulci asymmetries in chimpanzees and macaques: a new look at an old idea. Neuroimage 61:533-541. CrossRef Medline

Cantalupo C, Hopkins WD (2001) Asymmetric Broca's area in great apes. Nature 414:505. CrossRef Medline

Cantalupo C, Pilcher DL, Hopkins WD (2003) Are planum temporale and sylvian fissure asymmetries directly related? A MRI study in great apes. Neuropsychologia 41:1975-1981. CrossRef Medline

Cantalupo C, Oliver J, Smith J, Nir T, Taglialatela JP, Hopkins WD (2009) The chimpanzee show human-like perisylvian asymmetries in white matter. Eur J Neurosci 30:431-438. CrossRef Medline

Changizi MA (2001) Principles underlying mammalian neocortical scaling. Biol Cybern 84:207-215. CrossRef Medline

Clarkson MJ, Cardoso MJ, Ridgway GR, Modat M, Leung KK, Rohrer JD, Fox NC, Ourselin S (2011) A comparison of voxel and surface based cortical thickness estimation methods. Neuroimage 57:856-865. CrossRef Medline

Corballis MC (1992) The lopsided brain: evolution of the generative mind. New York: Oxford UP.

Crow TJ (2010) A theory of the origin of cerebral asymmetry: epigenetic variation superimposed on a fixed right-shift. Laterality 15:289-303. Medline

Das SR, Avants BB, Grossman M, Gee JC (2009) Registration based cortical thickness measurement. Neuroimage 45:867-879. CrossRef Medline 
Deacon TW (1997) The symbolic species: the coevolution of language and the brain. New York: Norton and Company.

Giedd JN, Blumenthal J, Jeffries NO, Castellanos FX, Liu H, Zijenbos A, Paus T, Evans AC, Rapoport JL (1999) Brain development during childhood and adolescence: a longitudinal study. Nat Neurosci 2:861-863.

Gorrie CA, Waite PM, Rogers LJ (2008) Correlations between hand preference and cortical thickness in the secondary somatosensory (SII) cortex of the common marmoset, Callithrix jacchus. Behav Neurosci 122:1343-1351. Medline

Hogstrom LJ, Westlye LT, Walhovd KB, Fjell AM (2012) The structure of the cerebral cortex across adult life: age-related patterns of surface area, thickness and gyrification. Cereb Cortex. Advance online publication. doi:10.1093/cercor/bhs231. CrossRef Medline

Hopkins WD, Nir TM (2010) Planum temporale surface area and grey matter asymmetries in chimpanzees (Pan troglodytes): the effect of handedness and comparison within findings in humans. Behav Brain Res 208: 436-443. CrossRef Medline

Hopkins WD, Taglialatela JP, Meguerditchian A, Nir T, Schenker NM, Sherwood CC (2008) Gray matter asymmetries in chimpanzees as revealed by voxel-based morphometry. Neuroimage 42:491-497. CrossRef

Hopkins WD, Taglialatela JP, Nir T, Schenker NM, Sherwood CC (2010) A voxel-based morphometry analysis of white matter asymmetries in chimpanzees (Pan troglodytes). Brain Behav Evol 76:93-100. CrossRef Medline

Im K, Lee J, Shin YW, Kim IY, Kwon JS, Kim SI (2006) Gender difference analysis of cortical thickness in healthy young adults with surface-based methods. Neuroimage 31:31-38. CrossRef Medline

Keller SS, Roberts N, Hopkins W (2009) A comparative magnetic resonance imaging study of the anatomy, variability and asymmetry of Broca's area in the human and chimpanzee brain. J Neurosci 29:14607-14616. CrossRef Medline

Klein A, Andersson J, Ardekani BA, Ashbumer J, Avants B, Chiang M, Christensen GE, Collins L, Hellier P, Song JH, Jenkinson M, Lepage C, Rueckert D, Thompson P, Vercauteren T, Woods RP, Mann JJ, Parsey RV (2009) Evaluation of 14 nonlinear deformation algorithms applied to human brain MRI registration. Neuroimage 46:786-802. CrossRef Medline

Li L, Preuss TM, Rilling JK, Hopkins WD, Glasser MF, Kumar B, Nana R, Zhang X, Hu X (2010) Chimpanzee pre-central corticospinal system asymmetry and handedness: a diffusion magnetic reonance imaging study. PLoS One 5:e12886. Medline

Luders E, Narr KL, Thompson PM, Rex DE, Jancke L, Toga AW (2006a) Hemispheric asymmetries in cortical thickness. Cereb Cortex 16:12321238. Medline

Luders E, Narr KL, Thompson PM, Rex DE, Woods RP, Deluca H, Jancke L, Toga AW (2006b) Gender effects on cortical thickness and the influence of scaling. Hum Brain Mapp 27:314-324. CrossRef Medline

Lyn H, Pierre P, Bennett AJ, Fears S, Woods R, Hopkins WD (2011) Planum temporale grey matter asymmetries in chimpanzees (Pan troglodytes), vervet (Chlorocebus aethiops sabaeus), rhesus (Macaca mulatta) and bonnet (Macaca radiata) monkeys. Neuropsychologia 49:2004-2012. CrossRef Medline

McGrew WC, Marchant LF (1997) On the other hand: current issues in and meta-analysis of the behavioral laterality of hand function in non-human primates. Yearb Phys Anthropol 40:201-232.

Rabinowicz T, Dean DE, Petetot JM, de Courten-Myers GM (1999) Gender differences in the human cerebral cortex: More neurons in males, more processes in females. J Child Neurol 14:98-107. CrossRef Medline

Rilling JK (2006) Human and non-human primate brains: are they allometrically scaled versions of the same design? Evol Anthropol 15:65-77. CrossRef

Rilling JK, Insel TR (1999a) Differential expansion of neural projection systems in primate brain evolution. Neuroreport 10:1453-1459. CrossRef Medline

Rilling JK, Insel TR (1999b) The primate neocortex in comparative perspective using magnetic resonance imaging. J Hum Evol 37:191-223. CrossRef Medline
Rilling JK, Seligman RA (2002) A quantitative morphometric comparative analysis of the primate temporal lobe. J Hum Evol 42:505-533. CrossRef Medline

Rilling JK, Glasser MF, Jbabdi S, Andersson J, Preuss TM (2011) Continuity, divergence and the evolution of brain language pathways. Front Evol Neurosci 3:11. Medline

Rogers J, Kochunov P, Zilles K, Shelledy W, Lancaster J, Thompson P, Duggirala R, Blangero J, Fox PT, Glahn DC (2010) On the genetic architecture of cortical folding and brain volume in primates. Neuroimage 53: 1103-1108. CrossRef Medline

Roth G, Dicke U (2005) Evolution of brain and intelligence. Trends Cogn Sci 9:250-257. CrossRef Medline

Schenker NM, Hopkins WD, Spocter MA, Garrison AR, Stimpson CD, Erwin JM, Hof PR, Sherwood CC (2010) Broca's area homologue in chimpanzees (Pan troglodytes): probabilistic mapping, asymmetry and comparison to humans. Cereb Cortex 20:730-742. CrossRef Medline

Schoenemann PT (2006) Evolution of size and functional areas of the human brain. Annu Rev Anthropol 35:379-406. CrossRef

Schoenemann PT, Sheehan MJ, Glotzer LD (2005) Prefrontal white matter volume is disproportionately larger in humans than in other primates. Nat Neurosci 8:242-252. CrossRef Medline

Seldon HL (2005) Does brain white matter growth expand the cortex like a balloon $>$ hypothesis and consequences. Laterality 10:81-95. Medline

Semendeferi K, Damasio H (2000) The brain and its main anatomical subdivisions in living hominids using magnetic resonance imaging. J Hum Evol 38:317-332. Medline

Semendeferi K, Damasio H, Frank R, Van Hoesen GW (1997) The evolution of the frontal lobes: a volumetric analysis based on three dimensional reconstructions of the magnetic resonance scans of human and ape brains. J Hum Evol 32:375-388. Medline

Semendeferi K, Armstrong E, Schleicher A, Zilles K, Van Hoesen GW (1998) Limbic frontal cortex in Hominoids: a comparative study of area 13. Am J Phys Anthropol 106:129-155. CrossRef Medline

Semendeferi K, Armstrong E, Schleicher A, Zilles K, Van Hoesen GW (2001) Prefrontal cortex in humans and apes: a comparative study of area 10. Am J Phys Anthropol 114:224-241. Medline

Shaw P, Greenstein D, Lerch J, Clasen L, Lenroot R, Gogtay N, Evans A, Rapoport J, Giedd J (2006) Intellectual ability and cortical development in children and adolescents. Nature 440:676-679. CrossRef Medline

Sherwood CC, Hof PR (2007) The evolution of neuron types and cortical histology in apes and humans. In: The evolution of primate nervous systems. Evolution of nervous systems (Preuss TM, Kaas J, eds). Oxford: Academic.

Sherwood CC, Baurernfeind AL, Bianchi S, Raghanti MA, Hof PR (2012) Human brain evolution writ large and small. In: Progress in brain research (Hofman MA, Falk D, eds). New York: Elsevier.

Smith SM, Jenkinson M, Woolrich MW, Beckmann CF, Behrens TEJ, Johansen-Berg H, Bannister PR, De Luca M, Drobniak I, Flitney DE, Niazy R, Saunders J, Vickers J, Zhang Y, De Stafano N, Brady JM, Matthews PM (2004) Advances in functional and structural MR image analysis and implementation of FSL. Neuroimage 23 [Suppl 1]:S208-S219. Medline

Sowell ER, Thompson PM, Leonard CM, Welcome SE, Kan E, Toga AW (2004) Longitudinal mapping of cortical thickness and brain growth in normal children. J Neurosci 24:8223-8231. CrossRef Medline

Spocter MA, Hopkins WD, Garrison AR, Bauernfeind AL, Stimpson CD, Erwin JM, Hof PR, Sherwood CC (2010) Wernicke's area homolog in chimpanzees (Pan troglodytes): probabilistic mapping, asymmetry and comparison with humans. Proc Biol Sci 277:2165-2174. CrossRef Medline

Styner M, Knickmeyer R, Joshi S, Coe C, Short SJ, Gilmore J (2007) Automatic brain segmentation in rhesus monkeys. SpieEE 65122:L65121-L65128.

Van Essen DC, Glasser MF, Dierker DL, Harwell J, Coalson T (2012) Parcellations and hemispheric asymmetries of human cerebral cortex analyzed on surface-based atlases. Cereb Cortex 22:2241-2262. CrossRef Medline 